\title{
Cooperative Innovation in the Medical Supply Chain Based on User Feedback
}

\author{
Ran Chen, Gui-sheng Hou, and Yu Wang \\ School of Economics and Management, Shandong University of Science and Technology, Qindao 266590, China \\ Correspondence should be addressed to Yu Wang; yuwang17@sdust.edu.cn
}

Received 17 February 2020; Revised 17 March 2020; Accepted 30 March 2020; Published 21 April 2020

Guest Editor: Lei Xie

Copyright ( $\odot 2020$ Ran Chen et al. This is an open access article distributed under the Creative Commons Attribution License, which permits unrestricted use, distribution, and reproduction in any medium, provided the original work is properly cited.

\begin{abstract}
Given the importance of users in medical innovation, positive user participation can boost the cooperative innovation process within the medical supply chain. A stochastic differential model based on user feedback is proposed to study the relationship between user feedback and the medical supply chain. The stability and sensitivity of the medical supply chain is analysed through different parameters. The results show that the effect of patient feedback and suggestions from hospitals on the innovation level of medical services and medical products is positive, such that the impact of the innovation level of medical services on users and the effect of patient feedback are positively related to marginal profits and that cooperative innovation is beneficial for medical product and service innovation and the improvement of demand and profits.
\end{abstract}

\section{Introduction}

With economic growth and increasing health awareness, demand for and spending on advanced medical products and services have increased due to the pursuit of longer lifespans and better living conditions $[1,2]$. Consumers are increasingly willing to pay more for advanced medical products and services [3].

Medical manufacturers develop and produce innovative medical products to meet this diverse and complex demand. Medical products include medicines, medical devices, and other supplementary medical products. Medical devices are necessary for patient recovery and physical rehabilitation. In most countries, the majority of medical device companies is small and medium-sized enterprises (SMEs); in the Netherlands, for example, the ratio of SMEs in this sector is approximately $80 \%$ [4]. The main market for their products is domestic. These companies have few opportunities to attract large investments, and it is difficult to launch new medical products owing to regulatory restrictions and licensing systems $[5,6]$. High technological capacity and innovative capacity are two main drivers of competition in the medical industry. Cooperating with other firms in this industry has been suggested due to the uncertainty involved in the technological innovation process, hysteresis, and revenue leakages [5]. Cooperation enables firms to earn more profits [7]. Cooperation in medical technological innovation is vital for companies that have a heavy dependence on the import of technology and advanced products. Alliances among enterprises, universities, and research institutes play an important role in decreasing research risk and speeding up the identification of medical innovation breakthroughs. Thus, appropriate medical products and services can be developed in a shorter time [8]. Cooperation among industry, academia, hospitals, and patients is beneficial for the effective integration of various resources, the construction of an expansive knowledge network for medical innovation [9], and the development of open innovation platforms [10]. Patients not only are those who need a cure but also are consumers of medical products and services [11]. As a key knowledge source and the main consumers of such products and services, patients help lead long-term medical innovation and drive the primary trend of the market [12]. Users provide new ideas to companies and enable them to understand real demands and expectations, reduce costs, develop product design and safety, and identify potential problems with medical products in the development stage $[13,14]$. Users also offer real-time 
feedback after the introduction of new products in the market [12]. However, companies must involve users in product development at the right time using the right methods to make the cooperation work [15].

Users include both patients and hospitals. For hospitals, the aim of medical services is the promotion of the public good instead of profit maximum [16]. Hospital managers need to consider effectiveness, patient safety, budget, treatment requirements, reputation, and cost in developing a hospital's medical technology strategy [17]. The hospital cooperates with medical suppliers, provides medical services, and collects patient information and feedback. An adequate understanding of patient needs and effective interaction and communication with patients during the process of supplying medical services are indispensable for increasing the quality of medical services. Feedback from patients on their own diseases and relevant treatments has high heterogeneity and direction [18]. Physician participation is beneficial for building medical information systems to gather more accurate information [19]. Surgeons are receptive to the marketing of medical products and to patient feedback [20]. While some elements of patient feedback are characterized by low credibility, communication between medical companies and patients has some impact on the value of feedback [21]. To improve feedback credibility, most medical companies prefer to offer commissions to hospitals. The authors in [3] point out that the commission rate is dependent upon hospitals' effort level.

The identification of unmet and unrecognized needs [22], interaction with patients and their families, and an emphasis on patient feedback are critical to the construction of a patient-oriented healthcare system [23]. The incentives to further these objectives should be relevant, practical, and sustainable [24]. Governments often make some efforts: the British government, for example, has established healthcare technology cooperatives to boost cooperation among hospitals, patients, industry, and academia to develop new products and update existing ones [25]. In addition, in many countries, excellent licensing, technology evaluation, and payment systems have been established [26]. Brazil's government has passed incentives to enhance cooperation between universities and public medical institutes [27]. In other countries, green approval channels have been setup for some medical devices and medicine.

Currently, only a few medical companies prefer to collect suggestions extensively at the concept design stage, and a small number of users are invited to participate in the development and testing stages [28]. Most companies do not invite users to join in the product design and development phases [20] and ignore the significant impacts of user participation. Companies that do want to bring users into their development system need to take into account how user participation will affect product innovation, how to promote the positive role of users and hospitals in cooperative medical innovation, and how to deal with the impacts of the inherent randomness of the market and consumer choice using optimal strategies. In this paper, a strategic model is constructed based on stochastic differential game theory to solve the problems mentioned above. The model is chosen due to the following benefits: compared with other strands of game theory, stochastic differential theory allows the randomness in the process and uncertainty during the decision-making process to be approached effectively and fits the real world better. The theory has been widely applied in the fields of economics and operations research. In [29], the authors find strategies with reduced risks by using stochastic differential game theory. The authors in [30] study cooperation in green building technology by using stochastic differential game theory to model the uncertainty of the external environment. The current research aims to promote cooperation among users, hospitals, and companies based on users' feedback and hospitals' suggestions, while considering uncertainty.

The contribution of this study is to group feedback into two types-patient feedback and hospital suggestions-and analyse the impacts of both types on the optimal strategies for cooperative innovation and for the innovation partners. Uncertainty of choice and the environment is considered to reflect the real world.

This study is organized into six parts as follows. Section 1 introduces the background. The stochastic differential model is constructed in Section 2. Sections 3 and 4 present the process of model solving. Finally, the discussion, numerical simulations, and conclusion can be found in Sections 5 and 6.

\section{Proposed Stochastic Differential Model}

A two-echelon supply chain has been widely applied in related research [31-33]. Here, the supply chain includes a medical manufacturer and a hospital. The medical manufacturer is the leader and responsible for the supply of medical products. The hospital is the follower and provides the corresponding medical services and treatment for patients; both players are risk neutral. The medical manufacturer is dominant in the market and sets monopoly prices to attain profits $[1,34,35]$. The manufacturer makes development decisions based on feedback from the hospital. Innovation in the medical supply chain can include the development of new medical products and medical services and treatments simultaneously. The medical manufacturer decides the wholesale price of new products and the innovation level, and the hospital decides the retail price. The conceptual model is shown in Figure 1.

Considering the existing research $[3,36,37]$, the cost functions of the medical manufacturer and the hospital are set as

$$
\begin{aligned}
C_{s}(L) & =\frac{\alpha}{2\left(1+\chi_{2}\right)} L^{2}(t), \\
C_{h}(G) & =\frac{\beta}{2\left(1+\chi_{1}\right)} G^{2}(t),
\end{aligned}
$$

where $\alpha>0$ and $\beta>0$ are the cost-effectiveness of the medical products and services, respectively; $L(t)$ and $G(t)$ are the innovation levels of the medical products and services at time $t$, respectively; and $\chi_{1}>0 \quad \chi_{2}>0$ are the 


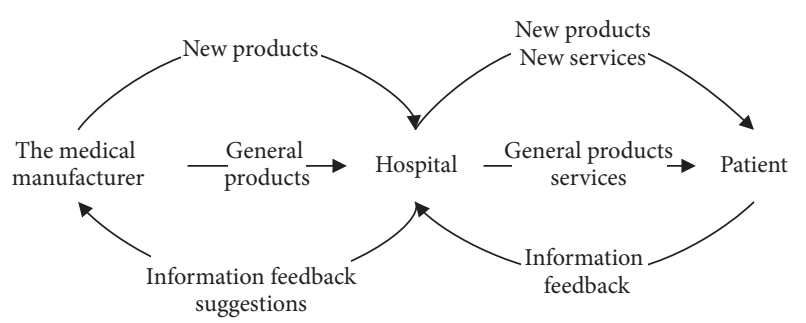

Figure 1: The concept of feedback in the medical supply chain innovation process.

effectiveness of the feedback from patients and the hospital, respectively.

When demand is unknown, there are some methods to describe the demand function, such as the exponential smoothing algorithm [38]. In this work, users purchase medical products and services based on the innovation level. That is, demand is a stochastic process that depends on the innovation level of medical products and services:

$$
\left\{\begin{array}{l}
\mathrm{d} X(t)=[\delta L(t)+\gamma G(t)-\varepsilon X(t)] \mathrm{d} t+\sigma(X(t)) \mathrm{d} W(t) \\
X(0)=X_{0} \geq 0
\end{array}\right.
$$

where $X(t)$ is the demand at time $t, \delta$, and $\gamma$ are the impacts of medical products and services on demand, respectively, $\varepsilon>0$ is the demand error term, $W(t)$ is the standard Wiener process at time $t$, and $\sigma(X(t))$ is the random interference coefficient.

The medical manufacturer and the hospital share the profits based on a previous contract, whereby the medical manufacturer earns $n \in(0,1)$ and the hospital receives $1-n$. The medical manufacturer shares costs with the hospital in the ratio $j(t) \in(0,1)$, where $j(t)$ denotes the commission rate. Subsidy $I$ is paid by the government. The discount rate for the medical manufacturer and hospital is $\rho(\rho>0)$, and $t$ is omitted in the following functions. The profit functions of the whole medical supply chain $[39,40]$, the medical manufacturer, and the hospital are as follows:

$$
\begin{aligned}
\pi(t)= & p X(t), \\
\max _{L, j} J_{s}\left(X_{0}\right)= & E \int_{0}^{\infty} e^{-\rho t}\left\{n \pi(t)-\frac{\alpha}{2\left(1+\chi_{2}\right)} L^{2}(t)\right. \\
& \left.-j(t) \frac{\beta}{2\left(1+\chi_{1}\right)} G^{2}(t)+I\right\} \mathrm{d} t, \\
\max _{G} J_{h}\left(X_{0}\right)= & E \int_{0}^{\infty} e^{-\rho t}\{(1-n) \pi(t) \\
& \left.-[1-j(t)] \frac{\beta}{2\left(1+\chi_{1}\right)} G^{2}(t)\right\} \mathrm{d} t,
\end{aligned}
$$

where $p$ is the retail price.
TABLE 1: Nomenclature.

\begin{tabular}{lc}
\hline Symbol means & Government subsidy \\
\hline$I$ & Demand \\
$G$ & Innovation level of medical services \\
$p$ & Retail price \\
$\sigma$ & Random interference coefficient \\
$\varepsilon$ & Demand error \\
$W$ & Standard wiener process \\
$j$ & Commission rate \\
$\rho$ & Discount rate \\
$\alpha$ & Cost-effectiveness of the medical products \\
$L$ & Innovation level of new products \\
$\beta$ & Cost-effectiveness of medical services \\
$\chi_{1}$ & Effectiveness of feedback from patients \\
$\chi_{2}$ & Effectiveness of feedback from the hospital \\
$\delta$ & Impact of medical products on demand \\
$\gamma$ & Impact of medical services on demand \\
$n$ & Profit ratio for the medical manufacturer \\
\hline
\end{tabular}

All the variables used in the following model are listed in Table 1.

\section{Solving of the Model}

3.1. Decentralized Model. The medical manufacturer and the hospital maximize their profits, and the optimal strategy is

$$
\left\{\begin{array}{l}
G^{*}=\frac{2 \gamma\left(1+\chi_{1}\right)(1-n)^{2} p}{\beta(\rho+\varepsilon)(1+n)}, \\
L^{*}=\frac{\delta\left(1+\chi_{2}\right) n p}{\alpha(\rho+\varepsilon)}, \\
j^{*}=\frac{3 n-1}{n+1}, n \in\left(\frac{1}{3}, 1\right) .
\end{array}\right.
$$

Proof. Feedback control with better capacity is widely applied in the economy [41]. The optimal value functions are $V_{h}(X)$ and $V_{s}(X)$, which should satisfy the Hamilton-Jacobi-Bellman (HJB) function:

$$
\begin{aligned}
\rho V_{h}(X)= & \max _{G}\left\{(1-n) p X-(1-j) \frac{\beta}{2\left(1+\chi_{1}\right)} G^{2}\right. \\
& \left.+V_{h}^{\prime}(X)(\delta L+\gamma G-\varepsilon X)+\frac{\sigma^{2}(X)}{2} V_{h}^{\prime \prime}(X)\right\} \\
\rho V_{s}(X)= & \max _{L, j}\left\{n p X-\frac{\alpha}{2\left(1+\chi_{2}\right)} L^{2}-j \frac{\beta}{2\left(1+\chi_{1}\right)} G^{2}+I\right. \\
& \left.+V_{s}^{\prime}(X)(\delta L+\gamma G-\varepsilon X)+\frac{\sigma^{2}(X)}{2} V_{s}^{\prime \prime}(X)\right\}
\end{aligned}
$$

where $V_{h}^{\prime}(X)$ and $V_{h}^{\prime \prime}(X)$ are the first and second partial derivatives of $V_{h}(X)$ and $V_{s}^{\prime}(X)$ and $V_{s}^{\prime \prime}(X)$ are the first and second partial derivatives of $V_{s}(X)$. 
Then, solve equation (7) and insert it into equation (6) to achieve equation (8). Maximize equation (8) to obtain equations (9) and (10). Insert (5) and (6) to obtain equations (11) and (12).

$$
\begin{aligned}
G= & \frac{\gamma V_{h}^{\prime}(X)\left(1+\chi_{1}\right)}{(1-j) \beta}, \\
\rho V_{s}(X)= & \max _{L, j}\left\{n p X-\frac{\alpha}{2\left(1+\chi_{2}\right)} L^{2}-\frac{j\left(1+\chi_{1}\right) \gamma^{2} V_{h}^{\prime 2}(X)}{2(1-j)^{2} \beta}\right. \\
& +I+V_{s}^{\prime}(X)\left(\delta L+\frac{\gamma^{2}\left(1+\chi_{1}\right) V_{h}^{\prime}(X)}{(1-j) \beta}-\varepsilon X\right) \\
& \left.+\frac{\sigma^{2}(X)}{2} V_{s}^{\prime \prime}(X)\right\} \\
& +V_{s}(X)= \\
& \frac{\delta V_{s}^{\prime}(X)\left(1+\chi_{2}\right)}{\alpha}, \\
& +\frac{\gamma^{2}\left(1+\chi_{1}\right)\left[2 V_{s}^{\prime}(X)+V_{h}^{\prime}(X)\right]^{2}}{8 \beta} \\
& +\frac{\sigma_{s}^{\prime}(X)-V_{h}^{\prime}(X)}{2 V_{s}^{\prime}(X)+V_{h}^{\prime}(X)} \\
& \left.+\frac{\sigma^{2}(X)\left(1+\chi_{2}\right)}{2 \alpha}+\frac{\sigma^{2}(X)}{2} V_{s}^{\prime \prime}(X)\right\} \\
\rho V_{h}(X)= & \max _{G}\left\{\left[(1-n) p-\varepsilon V_{h}^{\prime}(X)\right] X\right. \\
\alpha &
\end{aligned}
$$

Set $V_{h}(X)=f_{1} X+f_{2}$ and $V_{s}(X)=k_{1} X+k_{2}$, which are denoted by the optimal linear functions, where $f_{1}, f_{2}, k_{1}$, and $k_{2}$ are constants. Insert them into (11) and (12) to obtainfd 15

$$
\left\{\begin{array}{l}
f_{1}=\frac{(1-n) p}{\rho+\varepsilon} \\
f_{2}=\frac{\delta^{2}\left(1+\chi_{2}\right)(1-n)^{2} p^{2}}{\alpha \rho(\rho+\varepsilon)^{2}}+\frac{\gamma^{2}\left(1+\chi_{1}\right)\left(1-n^{2}\right) p^{2}}{4 \beta \rho(\rho+\varepsilon)^{2}} \\
k_{1}=\frac{n p}{\rho+\varepsilon} \\
k_{2}=\frac{\gamma^{2}\left(1+\chi_{1}\right)(p+n p)^{2}}{8 \beta \rho(\rho+\varepsilon)^{2}}+\frac{\delta^{2}\left(1+\chi_{2}\right)(1-n)^{2} p^{2}}{2 \alpha \rho(\rho+\varepsilon)^{2}}+I .
\end{array}\right.
$$

The optimal value functions of the medical manufacturer and the hospital are

$$
\begin{aligned}
V_{h}(X)= & \frac{(1-n) p}{\rho+\varepsilon} X+\frac{\delta^{2}\left(1+\chi_{2}\right)(1-n)^{2} p^{2}}{\alpha \rho(\rho+\varepsilon)^{2}} \\
& +\frac{\gamma^{2}\left(1+\chi_{1}\right)\left(1-n^{2}\right) p^{2}}{4 \beta \rho(\rho+\varepsilon)^{2}}, \\
V_{s}(X)= & \frac{n p}{\rho+\varepsilon} X+\frac{\gamma^{2}\left(1+\chi_{1}\right)(p+n p)^{2}}{8 \beta \rho(\rho+\varepsilon)^{2}} \\
& +\frac{\delta^{2}\left(1+\chi_{2}\right)(1-n)^{2} p^{2}}{2 \alpha \rho(\rho+\varepsilon)^{2}}+I .
\end{aligned}
$$

Corollary 1. The innovation level of medical services is positively related to demand, the effectiveness of feedback from patients, the retail price, and the commission rate $\left((\partial G / \partial \gamma)>0,\left(\partial G / \partial \chi_{1}\right)>0,(\partial G / \partial p)>0,(\partial G / \partial j)>0\right)$. The more real and effective the feedback from patients, the better are the medical services. The cost and demand error are negatively related to the innovation level of medical services $((\partial G / \partial \beta)<0, \quad(\partial G / \partial \varepsilon)<0)$.

Corollary 2. The innovation level of medical products is positively related to demand, the effectiveness of feedback from the hospital, and the retail price $((\partial L / \partial \delta)>0$, $\left.\left(\partial L / \partial \chi_{2}\right)>0,(\partial L / \partial p)>0\right)$. The positive effects of the innovation level of medical products on demand are significant for the development of products. The cost and demand error are negatively related to the innovation level of medical products $((\partial L / \partial \alpha)<0, \quad(\partial L / \partial \varepsilon)<0)$.

Considering the method proposed in [42], the optimal strategy is inserted into (2) to obtain

$$
\left\{\begin{array}{l}
\mathrm{d} X(t)=[\Omega-\varepsilon X(t)] \mathrm{d} t+\sigma(X(t)) \mathrm{d} W(t), \\
X(0)=X_{0} \geq 0,
\end{array}\right.
$$

where $\Omega=\left(\delta^{2}\left(1+\chi_{2}\right)(1-n) p / \alpha(\rho+\varepsilon)\right)+\left(2 \gamma^{2}\left(1+\chi_{1}\right)\right.$ $\left.(1-n)^{2} p /(1+n) \beta(\rho+\varepsilon)\right)$ is constant. The higher the innovation level of medical products, the larger the $\Omega$. Set $\sigma(X(t)) \mathrm{d} W(t)=\sigma \sqrt{X} \mathrm{~d} W(t)$ to obtain

$$
X(t)=X(0)+\int_{0}^{t}[\Omega-\varepsilon X(s)] \mathrm{d} s+\int_{0}^{t} \sigma(X(s)) \mathrm{d} W(s) .
$$

The expectation of equation (16) is $E(X(t))=X(0)+$ $\int_{0}^{t}[\Omega-\varepsilon X(s)] d s$. The initial condition is $E(X(0))=X_{0}$.

$$
\begin{aligned}
E(X(t))= & \frac{\Omega}{\varepsilon}\left(1-e^{-\varepsilon t}\right)+e^{-\varepsilon t} X_{0} \\
= & {\left[\frac{\delta^{2}\left(1+\chi_{2}\right)(1-n) p}{\alpha(\rho+\varepsilon) \varepsilon}+\frac{\gamma^{2}\left(1+\chi_{1}\right)(1-n) p}{(1-j) \beta(\rho+\varepsilon) \varepsilon}\right] } \\
& \cdot\left(1-e^{-\varepsilon t}\right)+e^{-\varepsilon t} X_{0}, \\
\lim _{t \rightarrow \infty} E(X(t))= & \frac{\Omega}{\varepsilon} .
\end{aligned}
$$


Substitute the Itô function into equation (15) to obtain

$$
\left\{\begin{array}{l}
\mathrm{d} X^{2}(t)=\left[2 X(\Omega-\varepsilon X)+\sigma^{2} X\right] \mathrm{d} t+2 X \sigma \sqrt{X} \mathrm{~d} W(t), \\
X^{2}(0)=X_{0}^{2} \geq 0
\end{array}\right.
$$

Then, integrate equations (15) and (18) to obtain the following equation:

$$
\begin{aligned}
D[X(t)] & =\frac{\sigma^{2} e^{-2 \varepsilon t}\left(e^{-\varepsilon t}-1\right)\left[\left(e^{-\varepsilon t}-1\right) \Omega+2 \varepsilon X_{0}\right]}{2 \varepsilon^{2}}, \\
\lim _{t \rightarrow \infty} D[X(t)] & =\frac{\sigma^{2} \Omega}{2 \varepsilon^{2}} .
\end{aligned}
$$

3.2. Centralized Model. When the players plan to cooperate, the objective becomes the profit maximization of the overall medical supply chain. The profit function is

$$
\begin{aligned}
\max _{L, G} J_{\mathrm{HS}}\left(X_{0}\right)= & E \int_{0}^{\infty} e^{-\rho t}\left\{p X(t)-\frac{\alpha}{2\left(1+\chi_{2}\right)} L^{2}(t)\right. \\
& \left.-\frac{\beta}{2\left(1+\chi_{1}\right)} G^{2}(t)+I\right\} \mathrm{d} t(t) .
\end{aligned}
$$

The optimal strategy in cooperation is

$$
\left\{\begin{array}{l}
L_{\mathrm{HS}}^{*}=\frac{\delta\left(1+\chi_{2}\right) p}{\alpha(\rho+\varepsilon)}, \\
G_{\mathrm{HS}}^{*}=\frac{\gamma\left(1+\chi_{1}\right) p}{\beta(\rho+\varepsilon)} .
\end{array}\right.
$$

The optimal value function is

$$
V_{\mathrm{HS}}(X)=\frac{p}{\rho+\varepsilon} X+\frac{\delta^{2}\left(1+\chi_{2}\right) p^{2}}{2 \alpha \rho(\rho+\varepsilon)^{2}}+\frac{\gamma^{2}\left(1+\chi_{1}\right) p^{2}}{2 \beta \rho(\rho+\varepsilon)^{2}}+I
$$

Corollary 3. The innovation level of medical products and services is positively related to demand, the effectiveness of feedback from the hospital and patients, and the retail price $\left(\left(\partial G_{H S}^{*} / \partial \gamma\right)>0,\left(\partial G_{H S}^{*} / \partial \chi_{1}\right)>0,\left(\partial G_{H S}^{*} / \partial p\right)>0,\left(\partial L_{H S}^{*} / \partial \delta\right)>\right.$ $\left.0,\left(\partial L_{H S}^{*} / \partial \chi_{2}\right)>0,\left(\partial L_{H S}^{*} / \partial p\right)>0\right)$.

The same method proposed before is used to obtain the expectation, variance, and stable value in the centralized strategy:

$$
\begin{aligned}
E_{\mathrm{HS}}(X(t)) & =\frac{\mho}{\varepsilon}\left(1-e^{-\varepsilon t}\right)+e^{-\varepsilon t} X_{0}, \\
\lim _{t \rightarrow \infty} E_{\mathrm{HS}}(X(t)) & =\frac{\mho}{\varepsilon}, \\
D_{\mathrm{HS}}[X(t)] & =\frac{\sigma^{2} e^{-2 \varepsilon t}\left(e^{-\varepsilon t}-1\right)\left[\left(e^{-\varepsilon t}-1\right) \mho+2 \varepsilon X_{0}\right]}{2 \varepsilon^{2}}, \\
\lim _{t \rightarrow \infty} D_{\mathrm{HS}}[X(t)] & =\frac{\sigma^{2} \mho}{2 \varepsilon^{2}} .
\end{aligned}
$$

\section{Discussion}

Insight 1. The innovation level of medical products in a centralized strategy is higher than that in a decentralized strategy. The difference between the medical product innovation level in the centralized and decentralized strategies depends on the impacts of innovation on demand and the effectiveness of the feedback from the hospital:

$$
\begin{array}{r}
\Delta L=L_{\mathrm{HS}}^{*}-L^{*}=\frac{\delta\left(1+\chi_{2}\right)(1-n) p}{\alpha(\rho+\varepsilon)}>0, \\
\frac{\partial \Delta L}{\partial \delta}>0, \frac{\partial \Delta L}{\partial \chi_{2}}>0 .
\end{array}
$$

Insight 2. The innovation level of medical services in a centralized strategy is higher than that in a decentralized strategy. The difference between medical service innovation level in the centralized and decentralized strategies depends on the impacts of innovation on demand and the effectiveness of the feedback from patients:

$$
\begin{aligned}
& \Delta G=G_{\mathrm{HS}}^{*}-G^{*}=\frac{\gamma\left(1+\chi_{1}\right) p\left[(1+n)-2(1-n)^{2}\right]}{\beta(\rho+\varepsilon)(1+n)}, \\
& n \in\left(\frac{1}{3}, 1\right), \Delta G>0, \frac{\partial \Delta G}{\partial \gamma}>0 \frac{\partial \Delta G}{\partial \chi_{1}}>0 .
\end{aligned}
$$




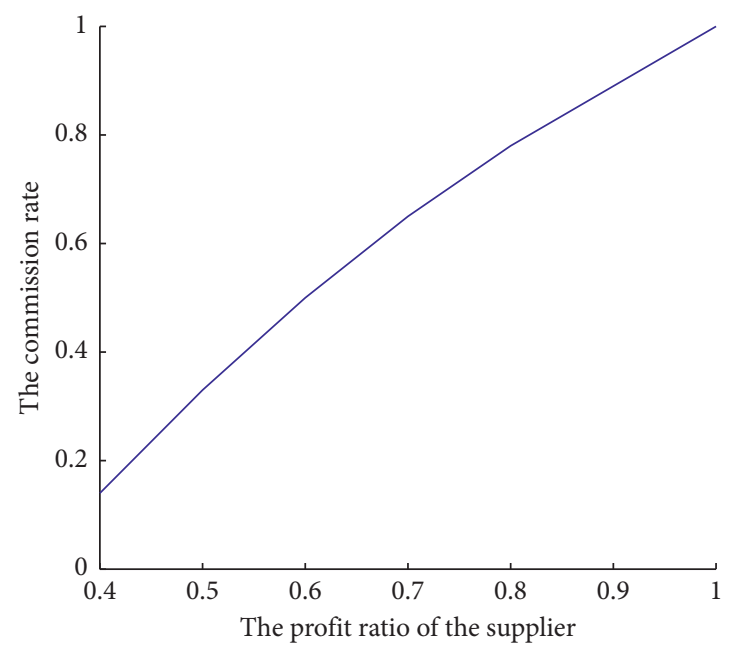

Figure 2: The commission rate in the decentralized strategy.

Insight 3. When $n \in((4 \sqrt{5} / 5)-1,1)$, profits are higher in the centralized strategy than in the decentralized strategy:

$$
\begin{aligned}
& \Delta V= V_{\mathrm{HS}}(X)-\left[V_{h}(X)+V_{s}(X)\right] \\
&= \frac{\delta^{2}\left(1+\chi_{2}\right) p^{2}\left(1-3(1-n)^{2}\right)}{2 \alpha \rho(\rho+\varepsilon)^{2}} \\
&+\frac{\gamma^{2}\left(1+\chi_{1}\right) p^{2}\left(5 n^{2}-11-10 n\right)}{8 \beta \rho(\rho+\varepsilon)^{2}}, \\
& n \in\left(\frac{4 \sqrt{5}}{5}-1,1\right), \quad \Delta V>0 .
\end{aligned}
$$

Insight 4. The expectation and variance of the innovation level of medical products and services and their stable values are higher in the centralized strategy than in the decentralized strategy:

$$
\begin{aligned}
\because \mho-\Omega & =\frac{n \delta^{2}\left(1+\chi_{2}\right) p}{\alpha(\rho+\varepsilon)}+\frac{\gamma^{2}\left(1+\chi_{1}\right) p(1-n)^{3}}{\beta(\rho+\varepsilon)(n+1)^{2}}>0, \\
\therefore E_{\mathrm{HS}}(X(t))-E(X(t)) & =\frac{\mho-\Omega}{\varepsilon}\left(1-e^{-\varepsilon t}\right)>0, \\
\lim _{t \longrightarrow \infty} E_{\mathrm{HS}}(X(t))-\lim _{t \rightarrow \infty} E(X(t)) & =\frac{\mho-\Omega}{\varepsilon}>0, \\
D_{\mathrm{HS}}(X(t))-D(X(t))=D_{\mathrm{HS}}[X(t)] & =\frac{\sigma^{2} e^{-2 \varepsilon t}\left(e^{-\varepsilon t}-1\right)^{2}(\mho-\Omega)}{2 \varepsilon^{2}}>0, \\
\lim _{t \rightarrow \infty} D_{\mathrm{HS}}(X(t))-\lim _{t \rightarrow \infty} D(X(t)) & =\frac{\sigma^{2}(\mho-\Omega)}{2 \varepsilon^{2}}>0 .
\end{aligned}
$$

\section{Numerical Simulation}

5.1. Optimal Strategy of the Medical Manufacturer and the Hospital. Set $\gamma=0.7, \delta=0.8, \alpha=5, \beta=4, \rho=0.8, \varepsilon=0.1$, and $p=10$ to study the impacts of user feedback and the share of profits on the optimal strategy.
Figures 2-4 give some information about the decentralized model. From Figure 2, the profits of the medical manufacturer are positively related to the commission rate. The more profits the medical manufacturer receives, the higher the commission rate the medical manufacturer offers. The positive impacts of feedback and suggestions from the 


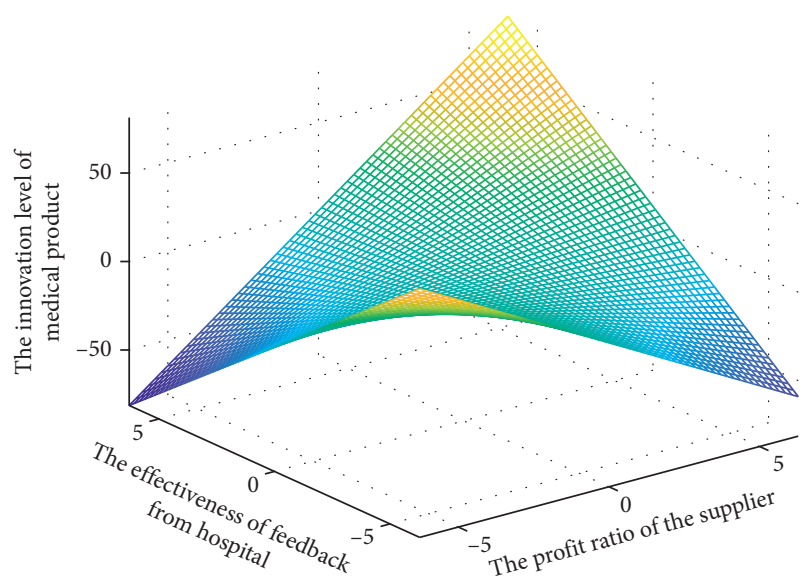

Figure 3: The innovation level of medical products in the decentralized strategy.

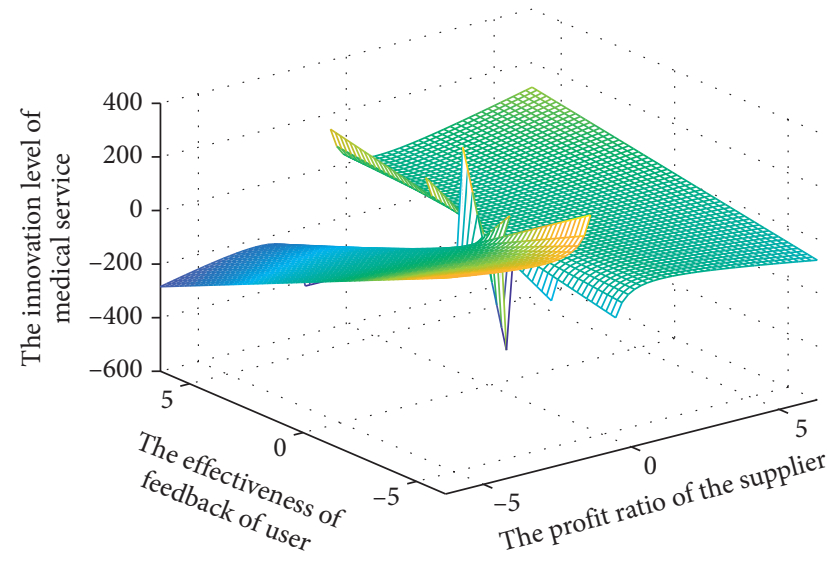

FIGURE 4: The innovation level of medical service in the decentralized strategy.

hospital and the profit ratio for the medical manufacturer on the innovation level of medical products can be found in Figure 3. That is, the real and effective information gathered and valuable suggestions supported by the hospital are beneficial for the improvement of medical products and meet the increasingly sophisticated demand. Figure 4 shows that there are positive impacts of feedback from patients and the profit ratio for the medical manufacturer on the innovation level of medical services. Though the hospital may receive lower profits, the commission makes up the gap. Figure 5 shows information about the positive impacts of the effectiveness of user feedback on the innovation level of medical products and services in the centralized strategy.

5.2. Effects of Demand and Demand Expectations. Set $\sigma=5$, $X_{0}=0, \chi_{1}=\chi_{2}=0.7, n=0.7$, and time step $\Delta t=1$. Based on the method presented in [42], (15) is discretized:

$$
X(t+\Delta t)=X(t)+(0.97-\varepsilon X(t)) \Delta t+\sigma \sqrt{X(t)} \sqrt{\Delta t} \zeta(t),
$$

where $\zeta(t)$ is the standard normal random variable.

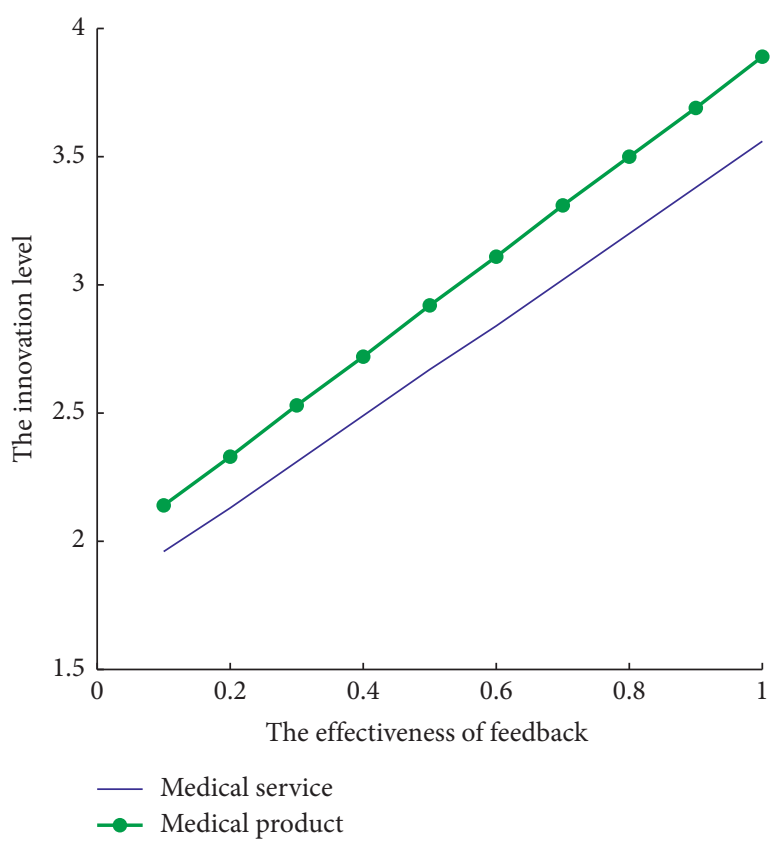

Figure 5: The impacts of feedback on the innovation level of medical products in the centralized strategy.

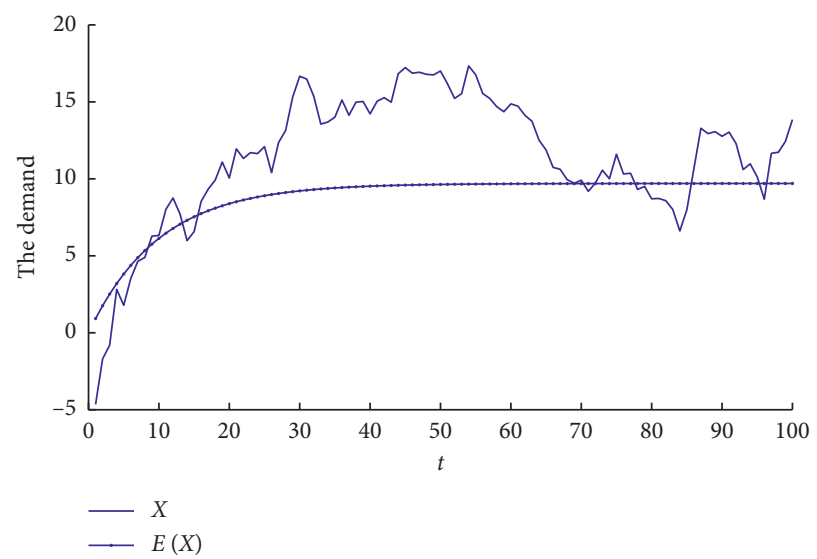

FIgURE 6: The changing effects of demand and demand expectations over time.

In Figure 6, demand fluctuates above and below its expected level. In real economies, demand is affected by various factors. It is difficult to obtain the actual data. The approximation of demand enables managers to make better decisions. Confidence intervals can describe real demand. The $95 \%$ confidence interval is

$$
(E[X(t)]-1.96 \sqrt{D[X(t)]}, E[X(t)]+1.96 \sqrt{D[X(t)]}) .
$$

\section{Conclusion}

Cooperation among medical manufacturers, medical service providers, and patients is fundamental to the construction of healthcare systems. In this paper, an innovation system 
involving patients, the medical manufacturer, and the hospital is established to allow for the consideration of user feedback. The medical manufacturer in this work is willing to collect suggestions from users and hospitals to achieve valuable feedback in the development and testing stage, highlighting the significant impacts of user participation. Stochastic differential game theory is used to find the optimal strategies in a decentralized and a centralized context. The results show that there are positive impacts of demand, the effectiveness of user feedback, and the retail price on the innovation level of medical products and services. User participation in the form of feedback has positive impacts on medical innovation. The cost and demand error term are negatively related to the innovation level of medical products and services. The innovation level of medical products and services is higher in the centralized strategy than in the decentralized strategy. It is possible to promote cooperation among users and hospitals in medical innovation by sharing the hospital's costs. The impacts of the inherent randomness of the market and consumer choice on the optimal strategies are described by the stochastic differential game model. The expectation and variance of the innovation level of medical products and services and their stable values are higher in the centralized strategy than in the decentralized strategy.

This study is beneficial in helping medical manufacturers adjust their commission rates to increase the effectiveness of user feedback, realize the importance of user feedback for cooperative innovation, and reduce costs. It is vital to build a positive cooperation cycle to boost medical products and service upgrades and updates.

Some limitations need to be addressed in the future. In real economies, there are more than two members in the medical supply chain, and the number of agents in the medical supply chain will be increased in the following study. Some other key factors, such as user feedback, need to be elaborated upon to further this study. Finally, enriching the cooperation design to reflect cooperation among industry, universities, research institutes, hospitals, and patients is another direction for future study.

\section{Data Availability}

All data, the models used during the study that appear in the submitted article, and the codes used to support the findings of this study are available from the corresponding author upon request.

\section{Conflicts of Interest}

The authors declare that there are no conflicts of interest regarding the publication of this paper.

\section{Acknowledgments}

Thanks are extended for the help from lecturer Lu Liu. This study was supported by the Shandong University of Science and Technology innovation programme (no. SDKDYC180110).

\section{References}

[1] A. M. Garber, C. I. Jones, and P. Romer, "Insurance and incentives for medical innovation," Forum for Health Economics \& Policy, vol. 9, no. 2, 2011.

[2] V. Peiffer, C. A. Yock, P. G. Yock, and J. B. Pietzsch, "Valuebased care: a review of key challenges and opportunities relevant to medical technology innovators," Journal of Medical Devices, Transactions of the ASME, vol. 13, no. 2, 2019.

[3] Y. J. Lu, Y. Q. Deng, and X. F. Xu, "Research on incentive mechanism of medical service supply chain under information asymmetry," Journal of UESTC (Social Sciences Edition), vol. 21, no. 5, pp. 88-95, 2019.

[4] A. J. J. Pullen, P. C. De Weerd-Nederhof, A. J. Groen, and O. A. M. Fisscher, "Open innovation in practice: goal complementarity and closed NPD networks to explain differences in innovation performance for SMEs in the medical devices sector," Journal of Product Innovation Management, vol. 29, no. 6, pp. 917-934, 2012.

[5] Y. Su, "Research on the cooperative innovation model of medical device industry in the processes of production-studyresearch-application based on information theory," Technology Intelligence Engineering, vol. 2, no. 2, pp. 34-44, 2016.

[6] M. Lee, S. Park, and K. S. Lee, "What are the features of successful medical device start-ups? Evidence from Korea," Sustainability, vol. 11, no. 7, pp. 1-17, 2019.

[7] Z. Li and Z. Q. Ma, "Research on benefit distribution of essential drugs supply chain in purchase pattern of medical insurance bidding," Industrial Engineering and Management, vol. 24, no. 5, pp. 64-71, 2019.

[8] L. Puslecki and M. Staszkow, "New cooperation modes: an opportunity for polish biotechnological clusters," Managing Global Transitions: International Research Journal, vol. 13, no. 2, pp. 171-188, 2015.

[9] K. de Jager, C. Chimhundu, T. Saidi, and T. S. Douglas, "Medical device innovation in South Africa: evolution of collaboration networks (2001-2013)," South African Journal of Industrial Engineering, vol. 30, no. 2, pp. 26-44, 2019.

[10] C. F. Daiberl, S. J. Oks, A. Roth, K. M. Möslein, and S. Alter, "Design principles for establishing a multi-sided open innovation platform: lessons learned from an action research study in the medical technology industry," Electronic Markets, vol. 29, no. 4, pp. 711-728, 2019.

[11] F. Djellal and F. Gallouj, "Mapping innovation dynamics in hospitals," Research Policy, vol. 34, no. 6, pp. 817-835, 2005.

[12] C. Lettl, C. Hienerth, and H. G. Gemuenden, "Exploring how lead users develop radical innovation: opportunity recognition and exploitation in the field of medical equipment technology," IEEE Transactions on Engineering Management, vol. 55, no. 2, pp. 219-233, 2008.

[13] S. Ghulam, S. Shah, and I. Robinson, "Benefits of and barriers to involving users in medical device technology development and evaluation," International Journal of Technology Assessment in Health Care, vol. 23, no. 1, pp. 131-137, 2007.

[14] S. G. S. Shah, I. Robinson, and S. Alshawi, "Developing medical device technologies from users' perspectives: a theoretical framework for involving users in the development process," International Journal of Technology Assessment in Health Care, vol. 25, no. 4, pp. 514-521, 2009.

[15] C. Lettl, "User involvement competence for radical innovation," Journal of Engineering and Technology Management, vol. 24, no. 1-2, pp. 53-75, 2007. 
[16] L. Y. Gao and X. L. Wang, "Study on coordination in medical service supply chains with social benefit under revenue sharing contract," Soft Science, vol. 32, no. 8, pp. 91-97, 2018.

[17] S. Gurtner, "Making the right decisions about new technologies," Health Care Management Review, vol. 39, no. 3, pp. 245-254, 2014.

[18] D. Lee, "A model for designing healthcare service based on the patient experience," International Journal of Healthcare Management, vol. 12, no. 3, pp. 180-188, 2017.

[19] S. Martikainen, J. Viitanen, M. Korpela, and T. Lääveri, "Physicians' experiences of participation in healthcare IT development in Finland: willing but not able," International Journal of Medical Informatics, vol. 81, no. 2, pp. 98-113, 2012.

[20] A. G. Money, J. Barnett, J. Kuljis, M. P. Craven, J. L. Martin, and T. Young, "The role of the user within the medical device design and development process: medical device manufacturers' perspectives," BMC Medical Informatics and Decision Making, vol. 11, no. 1, 2011.

[21] M. P. Manary, W. Boulding, R. Staelin, and S. W. Glickman, "The patient experience and health outcomes," New England Journal of Medicine, vol. 368, no. 3, pp. 201-203, 2013.

[22] A. D. McCarthy, L. Sproson, O. Wells, and W. Tindale, "Unmet needs: relevance to medical technology innovation?" Journal of Medical Engineering \& Technology, vol. 39, no. 7, pp. 382-387, 2015.

[23] K. Luxford, D. G. Safran, and T. Delbanco, "Promoting patient-centered care: a qualitative study of facilitators and barriers in healthcare organizations with a reputation for improving the patient experience," International Journal for Quality in Health Care, vol. 23, no. 5, pp. 510-515, 2011.

[24] P. Lehoux, B. Williams-Jones, F. Miller, D. Urbach, and S. Tailliez, "What leads to better health care innovation? Arguments for an integrated policy-oriented research agenda," Journal of Health Services Research \& Policy, vol. 13, no. 4, pp. 251-254, 2008.

[25] D. N. M. Heron and P. W. B. Tindale Obe, "Healthcare technology co-operatives: innovative about innovation," Journal of Medical Engineering \& Technology, vol. 39, no. 7, pp. 378-381, 2015.

[26] Z. P. Ye, M. Tang, H. Y. Wang, and C. L. Jin, "The management system and payment framework of innovative medical technology in the United Kingdom," Chinese Health Resources, vol. 22, no. 4, pp. 321-325, 2019.

[27] M. P. Ryan, "Patent incentives, technology markets, and public-private bio-medical innovation networks in Brazil," World Development, vol. 38, no. 8, pp. 1082-1093, 2010.

[28] W. G. Biemans, "User and third-party involvement in developing medical equipment innovations," Technovation, vol. 11, no. 3, pp. 163-182, 1991.

[29] S. Mataramvura and B. Øksendal, "Risk minimizing portfolios and HJBI equations for stochastic differential games," Stochastics, vol. 80, no. 4, pp. 317-337, 2008.

[30] S. Yin and B. Li, "Transferring green building technologies from academic research institutes to building enterprises in the development of urban green building: a stochastic differential game approach," Sustainable Cities and Society, vol. 39, pp. 631-638, 2018.

[31] Y. Tian, J. H. Ma, and W. D. Lou, "Research on supply chain stability driven by consumer's channel preference based on complexity theory," Complexity, vol. 2018, Article ID 7812784, 13 pages, 2018.

[32] Q. Li, Y. Zhang, and Y. Huang, "The complexity analysis in dual-channel supply chain based on fairness concern and different business objectives," Complexity, vol. 2018, no. 2, pp. 1-13, 2018.

[33] Q. Li, X. Chen, Y. Huang, H. Gui, and S. Liu, "The impacts of green innovation input and channel service in a dual-channel value chain," International Journal of Environmental Research and Public Health, vol. 16, no. 22, p. 4566, 2019.

[34] J. Ma and Z. Guo, "The influence of information on the stability of a dynamic Bertrand game," Communications in Nonlinear Science and Numerical Simulation, vol. 30, no. 1-3, pp. 32-44, 2016.

[35] J. H. Ma and Z. B. Guo, "Implications for firms with limited information to take advantage of reference price effect in competitive settings," Complexity, vol. 2017, Article ID 9712626, 16 pages, 2017.

[36] J. G. Du, P. P. Dou, and L. W. Zhao, “The impact of retailer's risk aversion and fairness preference on green supply chain operation," Journal of Industrial Technological Economics, vol. 7, pp. 3-9, 2017.

[37] G. Hou, Y. Wang, and B. Xin, "A coordinated strategy for sustainable supply chain management with product sustainability, environmental effect and social reputation," Journal of Cleaner Production, vol. 228, no. 10, pp. 1143-1156, 2019.

[38] Q. Li, "The dynamic behaviors of a supply chain with stockdependent demand considering competition and deteriorating items," Kybernetes, vol. 45, no. 7, pp. 1109-1128, 2016.

[39] Y. M. Huang, Q. X. Li, and Y. H. Zhang, "The complexity analysis for price game model of risk-averse supply chain considering fairness concern," Complexity, vol. 2018, Article ID 9216193, 15 pages, 2018.

[40] Q. Li, X. Chen, and Y. Huang, "The stability and complexity analysis of a low-carbon supply chain considering fairness concern behavior and sales service," International Journal of Environmental Research and Public Health, vol. 16, no. 15, p. 2711, 2019.

[41] Y. Feng, "Fuzzy stochastic differential systems," Fuzzy Sets and Systems, vol. 115, no. 3, pp. 351-363, 2000

[42] A. Prasad and S. P. Sethi, "Competitive advertising under uncertainty: a stochastic differential game approach," Journal of Optimization Theory and Applications, vol. 123, no. 1, pp. 163-185, 2004. 